\title{
CONGRUENCES AND PRIME-PERSPECTIVITIES IN FINITE LATTICES
}

\author{
G. GRÄTZER
}

\begin{abstract}
In a finite lattice, a congruence spreads from a prime interval to another by a sequence of congruence-perspectivities through intervals of arbitrary size, by a 1955 result of J. Jakubík.

In this note, I introduce the concept of prime-perspectivity and prove the Prime-projectivity Lemma: a congruence spreads from a prime interval to another by a sequence of prime-perspectivities through prime intervals.

A planar semimodular lattice is slim if it contains no $\mathrm{M}_{3}$ sublattice. I introduce the Swing Lemma, a very strong version of the Prime-projectivity Lemma for slim, planar, semimodular lattices.
\end{abstract}

\section{INTRODUCTION}

To describe the congruence lattice, Con $L$, of a finite lattice $L$, note that a prime interval $\mathfrak{p}$ generates a join-irreducible congruence, $\operatorname{con}(\mathfrak{p})$, and conversely; see, for instance, the discussion on pages 213 and 214 of LTF (reference [10]). So if we can determine when $\operatorname{con}(\mathfrak{p}) \geq \operatorname{con}(\mathfrak{q})$ (that is, $\mathfrak{q}$ is collapsed by $\operatorname{con}(\mathfrak{p})$ ) holds for the prime intervals $\mathfrak{p}$ and $\mathfrak{q}$ of $L$, then we know the distributive lattice Con $L$ up to isomorphism.

This is accomplished by the following result of J. Jakubík 24] (see Lemma 238 in LTF), where $\Rightarrow$ is congruence-projectivity, see Section 2

Lemma 1. Let $L$ be a finite lattice and let $\mathfrak{p}$ and $\mathfrak{q}$ be prime intervals in $L$. Then $\mathfrak{q}$ is collapsed by $\operatorname{con}(\mathfrak{p})$ iff $\mathfrak{p} \Rightarrow \mathfrak{q}$.

Jakubík's condition can be visualized using Figure 2 we may have to go through intervals of arbitrary size to get from $\mathfrak{p}$ to $\mathfrak{q}$.

In this note, I introduce the concept of prime-perspectivity. Let $L$ be a finite lattice and let $\mathfrak{p}$ and $\mathfrak{q}$ be prime intervals of $L$. The first diagram in Figure 1 depicts $\mathfrak{p}$ down-perspective to $\mathfrak{q}$, in formula, $\mathfrak{p} \stackrel{\text { dn }}{\sim} \mathfrak{q}$. We define $\mathfrak{p}$ up-perspective to $\mathfrak{q}$, in formula, $\mathfrak{p} \stackrel{\text { up }}{\sim} \mathfrak{q}$, dually. In the second diagram in Figure $1, \mathfrak{q}$ is collapsed by $\operatorname{con}(\mathfrak{p})$, but we cannot get from $\mathfrak{p}$ to $\mathfrak{q}$ by down- and up-perspectivities.

We introduce a more general step: $\mathfrak{p}$ is prime-perspective down to $\mathfrak{q}$ if $\mathfrak{p}$ is downperspective to $\left[0_{\mathfrak{p}} \wedge 1_{\mathfrak{q}}, 1_{\mathfrak{q}}\right]$ and $\mathfrak{q}$ is contained in $\left[0_{\mathfrak{p}} \wedge 1_{\mathfrak{q}}, 1_{\mathfrak{q}}\right]$. In other words, we have an $N_{5}$ sublattice: $\left\{0_{\mathfrak{p}} \wedge 1_{\mathfrak{q}}, 0_{\mathfrak{p}}, 0_{\mathfrak{q}}, 1_{\mathfrak{q}}, 0_{\mathfrak{p}} \vee 0_{\mathfrak{q}}\right\}$, so $\mathfrak{p}$ is down-perspective to $\left[0_{\mathfrak{p}} \wedge 1_{\mathfrak{q}}, 1_{\mathfrak{q}}\right]$ and $\mathfrak{q}$ is contained in $\left[0_{\mathfrak{p}} \wedge 1_{\mathfrak{q}}, 1_{\mathfrak{q}}\right]$.

We formalize this as follows: the binary relation $\mathfrak{p}$ prime-perspective down to $\mathfrak{q}$, in formula, $\mathfrak{p} \stackrel{\mathrm{p}-\mathrm{dn}}{\longrightarrow} \mathfrak{q}$, is defined as $1_{\mathfrak{q}} \leq 1_{\mathfrak{p}}, \mathfrak{p} \stackrel{\text { dn }}{\sim}\left[0_{\mathfrak{q}}, 1_{\mathfrak{p}} \vee 0_{\mathfrak{q}}\right]$, and $0_{\mathfrak{p}} \wedge 1_{\mathfrak{q}} \leq 0_{\mathfrak{q}}$.

Date: July 20, 2022.

2010 Mathematics Subject Classification. Primary: 06B10.

Key words and phrases. Prime-perspective, congruence, congruence-perspective, perspective, prime interval. 

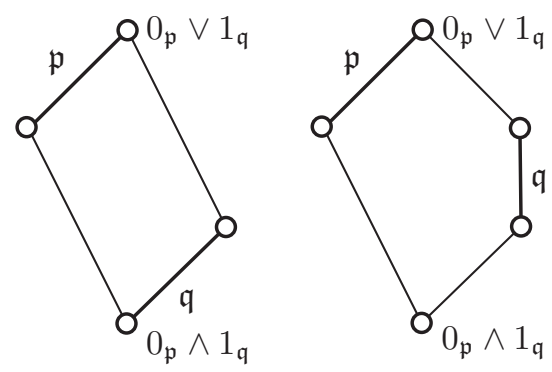

$$
\mathfrak{p} \stackrel{\mathrm{dn}}{\sim} \mathfrak{q} \quad \mathfrak{p} \stackrel{\mathrm{p}-\mathrm{dn}}{\longrightarrow} \mathfrak{q}
$$

FIGURE 1. Introducing prime-perspectivity

So if $\mathfrak{p} \stackrel{p-d n}{\longrightarrow} \mathfrak{q}$, then $\mathfrak{p}$ and $\mathfrak{q}$ generate an $N_{5}$, as in the second diagram of Figure 1 or a $\mathrm{C}_{2}^{2}$, as in the first diagram of Figure 1 , or $C_{2}$, if $\mathfrak{p}=\mathfrak{q}$.

We define prime-perspective up, $\mathfrak{p} \stackrel{\text { p-up }}{\longrightarrow} \mathfrak{q}$, dually. Let prime-perspective, $\mathfrak{p} \stackrel{p}{\longrightarrow} \mathfrak{q}$, mean that $\mathfrak{p} \stackrel{\text {-up }}{\longrightarrow} \mathfrak{q}$ or $\mathfrak{p} \stackrel{\text {-dn }}{\longrightarrow} \mathfrak{q}$ and let prime-projective, $\mathfrak{p} \stackrel{\mathrm{p}}{\longrightarrow} \mathfrak{q}$, be the transitive extension of $\stackrel{\mathrm{p}}{\longrightarrow}$.

Note 2. A prime-perspectivity $\mathfrak{p} \stackrel{\mathrm{p}-\mathrm{dn}}{\longrightarrow} \mathfrak{q}$ that is not a perspectivity is established by an $\mathrm{N}_{5}$ sublattice.
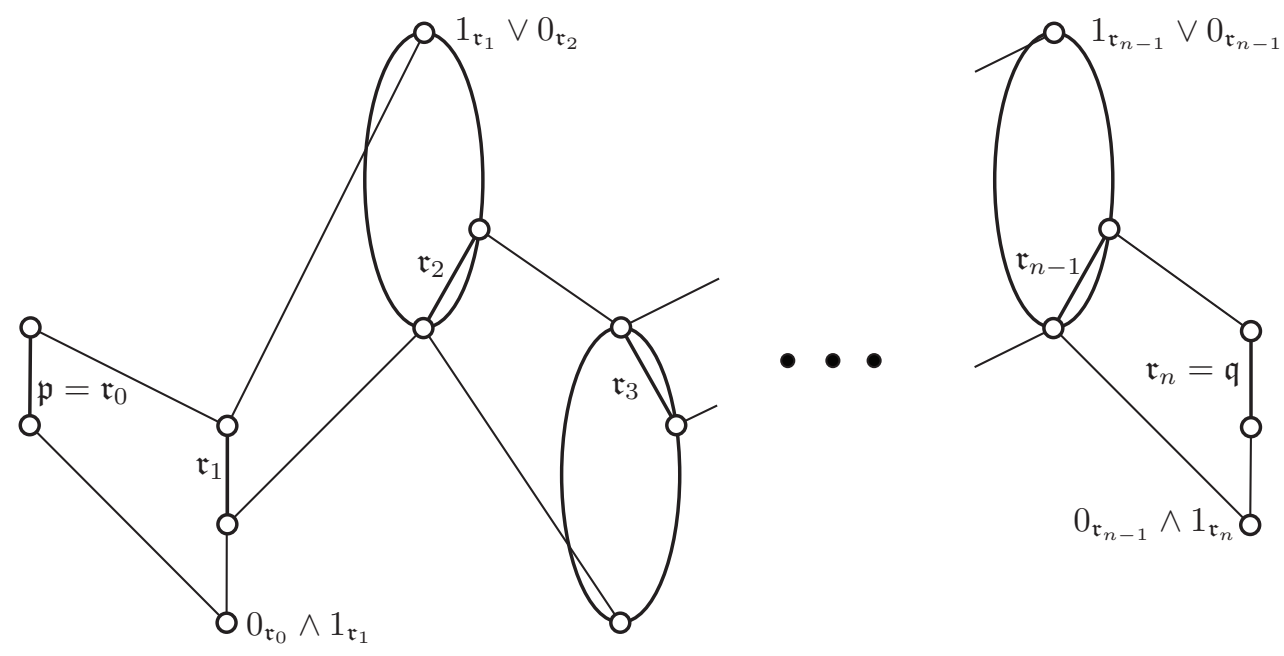

Figure 2. Prime-projectivity: $\mathfrak{p} \stackrel{p}{\Longrightarrow} \mathfrak{q}$

Note 3. The relation $\mathfrak{p} \stackrel{\mathrm{p}}{\longrightarrow} \mathfrak{q}$ holds iff the elements $\left\{0_{\mathfrak{p}}, 0_{\mathfrak{q}}, 1_{\mathfrak{p}}, 1_{\mathfrak{q}}\right\}$ satisfy a simple (universal) condition: $\mathfrak{p}$ and $\mathfrak{q}$ are perspective or they generate an $\mathrm{N}_{5}$ sublattice as in Figure 1

Note 4 . In the figures, bold lines designate coverings. 
Now we state our result: we only have to go through prime intervals to spread a congruence from a prime interval to another.

Lemma 5 (Prime-Projectivity Lemma). Let $L$ be a finite lattice and let $\mathfrak{p}$ and $\mathfrak{q}$ be distinct prime intervals in L. Then $\mathfrak{q}$ is collapsed by $\operatorname{con}(\mathfrak{p})$ iff $\mathfrak{p} \stackrel{p}{\Longrightarrow} \mathfrak{q}$, that is, iff there exists a sequence of pairwise distinct prime intervals $\mathfrak{p}=\mathfrak{r}_{0}, \mathfrak{r}_{1}, \ldots, \mathfrak{r}_{n}=\mathfrak{q}$ satisfying

$$
\mathfrak{p}=\mathfrak{r}_{0} \stackrel{p}{\longrightarrow} \mathfrak{r}_{1} \stackrel{p}{\longrightarrow} \cdots \stackrel{p}{\longrightarrow} \mathfrak{r}_{n}=\mathfrak{q} .
$$

Let us call a lattice $L$ an SPS lattice, if it is slim, planar, semimodular; see G. Grätzer and E. Knapp [18]. We state a strong form of the Prime-projectivity Lemma for SPS lattices, using the concept of a swing.

For the prime intervals $\mathfrak{p}, \mathfrak{q}$ of an SPS lattice $L$, we define a binary relation: $\mathfrak{p}$ swings to $\mathfrak{q}$, in formula, $\mathfrak{p} \in \mathfrak{q}$, if $1_{\mathfrak{p}}=1_{\mathfrak{q}}$, this element covers at least three elements, and $0_{\mathfrak{q}}$ is not the left-most or right-most element covered by $1_{\mathfrak{p}}=1_{\mathfrak{q}}$. We call the element $1_{\mathfrak{p}}=1_{\mathfrak{q}}$ the hinge of the swing.

See Figure 3 for two examples; in the first, the hinge covers three elements, in the second, five elements.
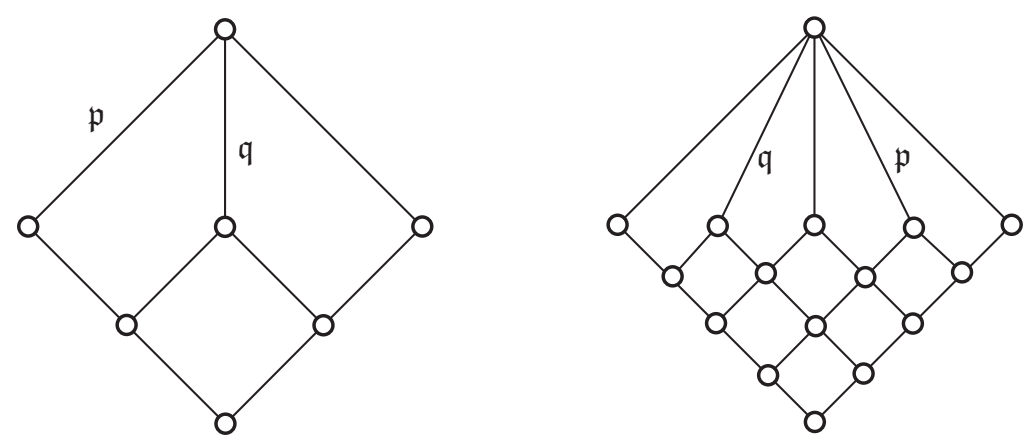

FiguRE 3. Swings, $\mathfrak{p} \backsim \mathfrak{q}$

Lemma 6 (Swing Lemma). Let $L$ be an SPS lattice and let $\mathfrak{p}$ and $\mathfrak{q}$ be distinct prime intervals in L. Then $\mathfrak{q}$ is collapsed by $\operatorname{con}(\mathfrak{p})$ iff there exists a prime interval $\mathfrak{r}$ and sequence of pairwise distinct prime intervals

$$
\mathfrak{r}=\mathfrak{r}_{0}, \mathfrak{r}_{1}, \ldots, \mathfrak{r}_{n}=\mathfrak{q}
$$

such that $\mathfrak{p}$ is up perspective to $\mathfrak{r}$, and $\mathfrak{r}_{i}$ is down perspective to or swings to $\mathfrak{r}_{i+1}$ for $i=0, \ldots, n-1$. In addition, the sequence (2) also satisfies

$$
1_{\mathfrak{r}_{0}} \geq 1_{\mathfrak{r}_{1}} \geq \cdots \geq 1_{\mathfrak{r}_{n}}
$$

The Swing Lemma is easy to visualize. Perspectivity up is "climbing", perspectivity down is "sliding". So we get from $\mathfrak{p}$ to $\mathfrak{q}$ by climbing once and then alternating sliding and swinging. In Figure 4 , we climb up from $\mathfrak{p}$ to $\mathfrak{r}=\mathfrak{r}_{0}$, swing from $\mathfrak{r}_{0}$ to $\mathfrak{r}_{1}$, slide down from $\mathfrak{r}_{1}$ to $\mathfrak{r}_{2}$, swing from $\mathfrak{r}_{2}$ to $\mathfrak{r}_{3}$, and finally slide down from $\mathfrak{r}_{3}$ to $\mathfrak{r}_{4}$.

Section 2 recalls some basic concepts and notation. In Section 3, I prove the Prime-projectivity Lemma. In Section 4 the Prime-projectivity Lemma is applied to verify a result of mine with E. Knapp [18]. Section 5 deals with the Swing Lemma. 


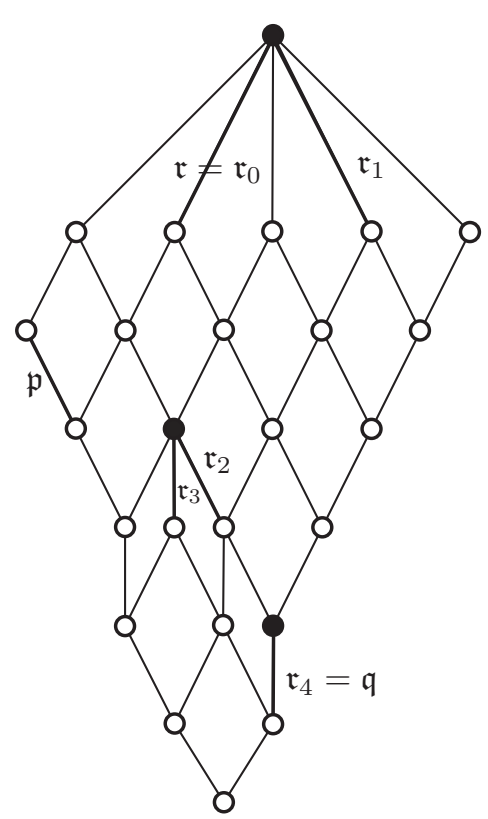

Figure 4. Illustrating the Swing Lemma

\section{Preliminaries}

We use the concepts and notation of LTF. For an ideal $I$, we use the notation $I=\left[00_{I}, 1_{I}\right]$.

We recall that $[a, b] \sim[c, d]$ denotes perspectivity, $[a, b] \stackrel{\text { up }}{\sim}[c, d]$ and $[a, b] \stackrel{\text { dn }}{\sim}[c, d]$ perspectivity up and down, see Figure $5 .[a, b] \approx[c, d]$ denotes projectivity, the transitive closure of perspectivity.

$[a, b] \rightarrow[c, d]$ denotes congruence-perspectivity, $[a, b] \stackrel{\text { up }}{\rightarrow}[c, d]$ and $[a, b] \stackrel{\mathrm{dn}}{\rightarrow}[c, d]$ denote congruence-perspectivity up and down, see Figure $6,[a, b] \Rightarrow[c, d]$ denotes congruence-projectivity, the transitive closure of congruence-perspectivity.

\section{Proving the Prime-Projectivity Lemma}

To prove the Prime-projectivity Lemma, let $\mathfrak{p}$ and $\mathfrak{q}$ be prime intervals in a finite lattice $L$ with $\operatorname{con}(\mathfrak{p}) \geq \operatorname{con}(\mathfrak{q})$. By Lemma 1, there is a sequence of congruenceperspectivities

$$
\mathfrak{p}=I_{0} \rightarrow I_{1} \rightarrow \cdots \rightarrow I_{m}=\mathfrak{q} .
$$

To get from (4) to (1), by induction on $m$, it is sufficient to prove the following statement.

Lemma 7. Let $L$ be a finite lattice and let $I \rightarrow J$ be intervals of $L$. Let $\mathfrak{b}$ be a prime interval in $J$. Then there exists a prime interval $\mathfrak{a} \subseteq I$ satisfying $\mathfrak{a} \stackrel{p}{\Longrightarrow} \mathfrak{b}$.

Proof. By duality, we can assume that $I \stackrel{\text { up }}{\leftrightarrow} J$. We prove the statement by induction on length $(I)$, the length of $I$ (that is, the length of the longest chain in $I$ ). Note that if $I^{\prime}$ is an interval properly contained in $I$, then length $\left(I^{\prime}\right)<\operatorname{length}(I)$.

For the induction base, let $I$ be prime. Then take $\mathfrak{a}=I$. 

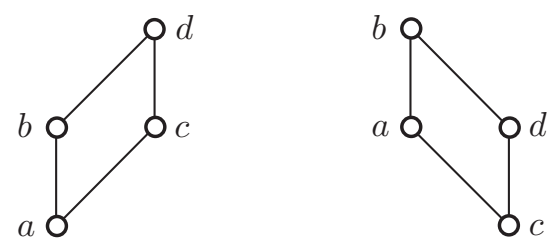

Figure 5. $[a, b] \sim[c, d]:[a, b] \stackrel{\text { up }}{\sim}[c, d]$ and $[a, b] \stackrel{\text { dn }}{\sim}[c, d]$
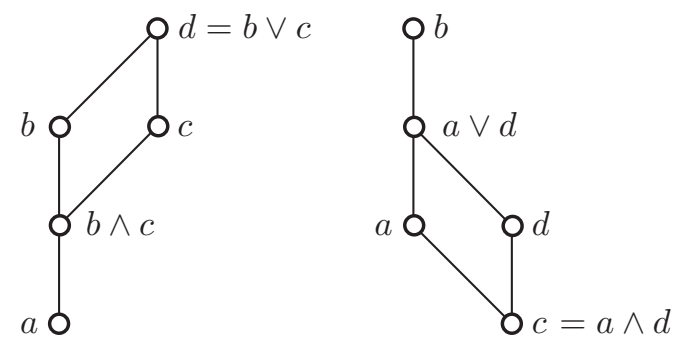

Figure $6 .[a, b] \stackrel{\text { up }}{\rightarrow}[c, d]$ and $[a, b] \stackrel{\mathrm{dn}}{\rightarrow}[c, d]$

For the induction step, we can assume that $I$ is not prime and that the statement is proved for intervals shorter than $I$.

Without loss of generality, we can assume that

(i) $J \nsubseteq I$-otherwise, take $\mathfrak{a}=\mathfrak{b} \subseteq I$;

(ii) $0_{\mathfrak{b}}=0_{J}$-otherwise, replace $J$ with $\left[0_{\mathfrak{b}}, 1_{J}\right]$;

(iii) $0_{I}=1_{I} \wedge 0_{\mathfrak{b}}$-otherwise, replace $I$ with $\left[1_{I} \wedge 0_{J}, 1_{I}\right] \subseteq I$.

Since $I$ is not a prime interval, there is an element $u \in I$ satisfying $0_{I} \prec u<1_{I}$. If $1_{\mathfrak{b}} \leq u \vee 0_{\mathfrak{b}}$, then take $\mathfrak{a}=\left[0_{I}, u\right]$; clearly, $\mathfrak{a} \stackrel{\text { p-up }}{\longrightarrow} \mathfrak{b}$.

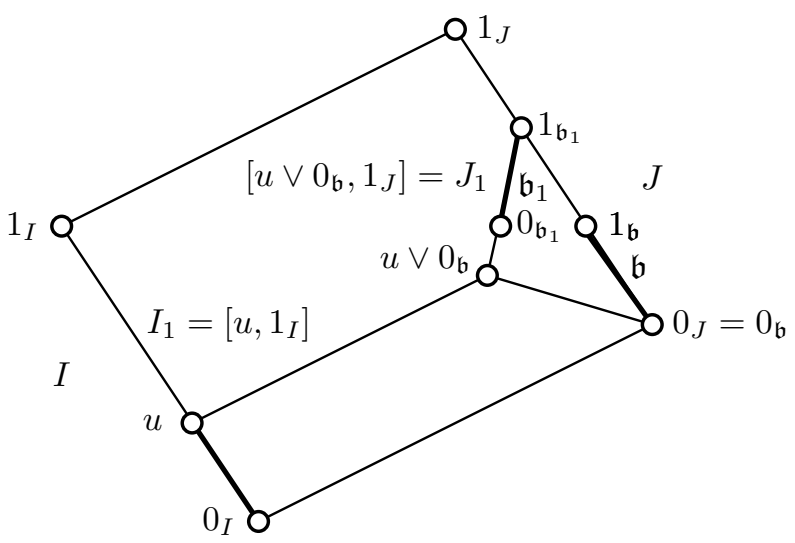

Figure 7. Proving the Prime-projectivity Lemma

Therefore, we can additionally assume that 
(iv) $u \vee 0_{\mathfrak{b}} \| 1_{\mathfrak{b}}$, see Figure 7. Then

$$
u \vee 1_{\mathfrak{b}}=\left(u \vee 0_{\mathfrak{b}}\right) \vee 1_{\mathfrak{b}}>u \vee 0_{\mathfrak{b}}
$$

So we can define a prime interval $\mathfrak{b}_{1}$ with

$$
u \vee 0_{\mathfrak{b}} \leq 0_{\mathfrak{b}_{1}} \prec 1_{\mathfrak{b}_{1}}=u \vee 1_{\mathfrak{b}} .
$$

Observe that

$$
\mathfrak{b}_{1} \stackrel{\mathrm{dn}}{\sim} \mathfrak{b}
$$

Applying the induction hypotheses to $I_{1}=\left[u, 1_{I}\right], J_{1}=\left[0_{\mathfrak{b}_{1}}, 1_{J}\right]$, and $\mathfrak{b}_{1}$, we obtain a prime interval $\mathfrak{a} \subseteq I_{1} \subseteq I$ satisfying $\mathfrak{a} \stackrel{\mathrm{p}}{\Longrightarrow} \mathfrak{b}_{1}$. Combining $\mathfrak{a} \stackrel{\mathrm{p}}{\Longrightarrow} \mathfrak{b}_{1}$ and (5), we obtain that $\mathfrak{a} \stackrel{\mathrm{p}}{\Longrightarrow} \mathfrak{b}$, as required.

The proof actually verified more than stated. Every step of the induction adds no more than one prime interval to the sequence.

\section{An applichtion}

In 18, I proved with E. Knapp the following result:

Theorem 8. Let $L$ be a finite lattice and let $\mathfrak{p}$ and $\mathfrak{q}$ be prime intervals in $L$ such that $\operatorname{con}(\mathfrak{p}) \succ \operatorname{con}(\mathfrak{q})$ in the order $\operatorname{Conj} L$ of join-irreducible congruences of $L$. Then there exist a sublattice $\mathrm{N}_{5}$ of $L$-depicted by Figure ?? - and prime intervals $\mathfrak{p}_{0}$ and $\mathfrak{q}_{0}$ in $\mathrm{N}_{5}$ satisfying $\operatorname{con}(\mathfrak{p})=\operatorname{con}\left(\mathfrak{p}_{0}\right)$ and $\operatorname{con}(\mathfrak{q})=\operatorname{con}\left(\mathfrak{q}_{0}\right)$.

Proof. Let $\mathfrak{p}$ and $\mathfrak{q}$ be prime intervals in $L$ such that $\operatorname{con}(\mathfrak{p}) \succ \operatorname{con}(\mathfrak{q})$ in the order Conj of join-irreducible congruences of $L$. By the Prime-projectivity Lemma, there exists prime intervals $\mathfrak{r}_{i}$, for $0 \leq i \leq m$, such that (1) holds. Clearly,

$$
\operatorname{con}(\mathfrak{p})=\operatorname{con}\left(\mathfrak{r}_{0}\right) \geq \operatorname{con}\left(\mathfrak{r}_{1}\right) \geq \cdots \geq \operatorname{con}\left(\mathfrak{r}_{m}\right)=\operatorname{con}(\mathfrak{q}) .
$$

So there is a (unique) $0 \leq k<m$ such that

$$
\begin{aligned}
\operatorname{con}\left(\mathfrak{r}_{k}\right) & =\operatorname{con}(\mathfrak{p}), \\
\operatorname{con}\left(\mathfrak{r}_{k+1}\right) & =\operatorname{con}(\mathfrak{q}) .
\end{aligned}
$$

Therefore, $\mathfrak{r}_{k}=\mathfrak{p}_{0}$ and $\mathfrak{r}_{k+1}=\mathfrak{q}_{0}$ satisfy the statement of Theorem 8 .

\section{The Swing Lemma}

5.1. Some basic concepts. For SPS lattices, we stated the Swing Lemma, a strong form of the Prime-projectivity Lemma. To discuss its proof, we need a few more concepts and results. (For a more detailed overview, see G. Czédli and G. Grätzer [5] and G. Grätzer 11.)

An element $c$ of an SPS lattice $L$ is a left corner, if it is a doubly-irreducible element on the left boundary of $L$ (excluding $0_{L}$ and $1_{L}$ ), its unique cover $c^{*}$ covers exactly two elements, and dually, for the lower cover $c_{*}$. We define a right corner symmetrically.

An SPS lattice $L$ is called rectangular if it has a unique left corner $c_{l}$ and a unique right corner $c_{r}$, and these two elements are complementary. See G. Grätzer and E. Knapp [16] 20] for these concepts and the two observations in the next paragraph.

Every SPS lattice $L$ has a congruence-preserving extension to a rectangular lattice $R$, which we obtain by adding corners. By the same token, every SPS lattice $L$ can be obtained from a rectangular lattice $R$ by removing corners. 
5.2. Verifying the Swing Lemma. We have two proofs of the Swing Lemma. G. Czédli applies in 2] the Trajectory Coloring Theorem for Slim Rectangular Lattices of G. Czédli [1, Theorem 7.3] to prove the Swing Lemma for rectangular lattices.

In view of the discussion at the end of Section 5.1. to complete the proof of the Swing Lemma, it is sufficient to prove the following result.

Lemma 9. Let $L$ be an SPS lattice. Let $c$ be a corner of $L$ and let $L^{\prime}=L-\{c\}$, a sublattice of $L$. Let us assume that the Swing Lemma holds for the lattice $L$. Then the Swing Lemma holds also for the lattice $L^{\prime}$.

Proof. Let $\mathfrak{p}$ and $\mathfrak{q}$ be prime intervals in $L^{\prime}$ such that $\mathfrak{q}$ is collapsed by $\operatorname{con}(\mathfrak{p})$ in $L^{\prime}$. Then we also have that $\mathfrak{q}$ is collapsed by $\operatorname{con}(\mathfrak{p})$ in $L$. Since the Swing Lemma is assumed to hold in $L$, therefore, there exists a sequence of pairwise distinct prime intervals (2) in $L$. We can assume that we choose the sequence in (2) so that $n$ and length $\left[1_{p}, 1_{r_{0}}\right]$ are minimal. To show that the Swing Lemma holds in $L^{\prime}$, we prove that every prime interval $\mathfrak{r}_{i}$ in the sequence (2) is in $L^{\prime}$.

Let $\left\{c, c_{*}, c^{*}, d\right\}$ be the (unique) covering square in $L$ containing $c$. The lattice $L$ has exactly two prime intervals not in $L^{\prime}$, namely, $\mathfrak{t}_{*}=\left[c_{*}, c\right]$ and $\mathfrak{t}^{*}=\left[c, c^{*}\right]$. We claim that neither appears in 22); the verification of this claim completes the proof of the lemma

Let us assume that $\mathfrak{t}^{*}$ appears in the sequence $(2)$ for $\mathfrak{p}$ and $\mathfrak{q}$. Since $c^{*}$ covers exactly two elements by the definition of a corner, it is not the hinge of a swing, so the step to $t^{*}$ must be a perspectivity up and the step from $t^{*}$ must be a perspectivity down; therefore, the sequence can be shortened, contradicting the minimality of $n$.

Let $\mathfrak{t}_{*}$ appear in the sequence. Since $c$ is doubly irreducible, it is not the hinge of a swing, so the step to $\mathfrak{t}_{*}$ must be a perspectivity down. For the same reason, the next step, back into $L^{\prime}$, must be a perspectivity up; therefore, the sequence can be shortened, contradicting again the minimality of $n$.

The second proof in G. Grätzer [14] is more "elementary"; it proves the Swing Lemma directly for SPS lattices.

\section{REFERENCES}

[1] G. Czédli, Patch extensions and trajectory colorings of slim rectangular lattices, Algebra Universalis 72 (2014), 125-154.

[2] _ A note on congruence lattices of slim semimodular lattices, Algebra Universalis.

[3] - From trajectory colorings to G. Grätzer's Swing Lemma in rectangular lattices.

[4] G. Czédli and G. Grätzer, Notes on planar semimodular lattices. VII. Resections of planar semimodular lattices. Order 29 (2012), 1-12.

[5] G. Czédli and G. Grätzer, Planar Semimodular Lattices: Structure and Diagrams. Chapter 4 in 23. 40 pp. plus bibliography.

[6] G. Czédli and E. T. Schmidt, The Jordan-Hölder theorem with uniqueness for groups and semimodular lattices, Algebra Universalis 66 (2011), 69-79.

[7] _ Slim semimodular lattices. I. A visual approach, Order 29 (2012), 481-497.

[8] Slim semimodular lattices. II. A description by patchwork systems, Order 30 (2013), 689-721.

[9] Composition series in groups and the structure of slim semimodular lattices, Acta Sci. Math. (Szeged) 79 (2013), 369-390.

[10] G. Grätzer, Lattice Theory: Foundation. Birkhäuser Verlag, Basel, 2011. xxix+613 pp. ISBN: 978-3-0348-0017-4.

[11] G. Grätzer, Planar Semimodular Lattices: Congruences. Chapter 4 in [23]. 32 pp. plus bibliography. 
[12] G. Grätzer, Congruences of fork extensions of lattices. Algebra Universalis. arXiv: 1307.8404

[13] - On a result of Gábor Czédli concerning congruence lattices of planar semimodular lattices. Acta Sci. Math. (Szeged).

[14] _ Congruences in slim, planar, semimodular lattices.

[15] G. Grätzer, H. Lakser, and E. T. Schmidt, Congruence lattices of finite semimodular lattices. Canad. Math. Bull. 41 (1998), 290-297.

[16] G. Grätzer and E. Knapp, Notes on planar semimodular lattices. I. Construction. Acta Sci. Math. (Szeged) 73 (2007), 445-462.

[17] _ A note on planar semimodular lattices. Algebra Universalis 58 (2008), 497-499.

[18] _ Notes on planar semimodular lattices. II. Congruences. Acta Sci. Math. (Szeged) 74 (2008), 37-47.

[19] , Notes on planar semimodular lattices. III. Rectangular lattices. Acta Sci. Math. (Szeged) 75 (2009), 29-48.

[20] _ Notes on planar semimodular lattices. IV. The size of a minimal congruence lattice representation with rectangular lattices. Acta Sci. Math. (Szeged) 76 (2010), 3-26.

[21] G. Grätzer and J. B. Nation, A new look at the Jordan-Hölder theorem for semimodular lattices. Algebra Universalis 64 (2011), 309-311.

[22] G. Grätzer and E. T. Schmidt, Ideals and congruence relations in lattices. Acta Math. Acad. Sci. Hungar. 9 (1958), 137-175.

[23] G. Grätzer and F. Wehrung eds., Lattice Theory: Special Topics and Applications. Volume 1. Birkhäuser Verlag, Basel, 2014.

[24] J. Jakubík, Congruence relations and weak projectivity in lattices, (Slovak) Časopis Pěst. Mat. 80 (1955), 206-216.

Department of Mathematics, University of Manitoba, Winnipeg, MB R3T 2N2, Canada E-mail address, G. Grätzer: gratzer@me.com

$U R L$, G. Grätzer: http://server.math.umanitoba.ca/homepages/gratzer/ 